\title{
Erotik als Teil Sozialer Arbeit
}

Verhältnisse zwischen Sozialarbeiter_innen und Klient_innen und unter Sozialarbeiter_innen

Warum wird Erotik in der Sozialen Arbeit so selten thematisiert, obwohl Erotik überall präsent ist, wo Menschen sich begegnen? Und wie verhält sich Erotik zu Liebe und Sexualität und welche Aufgaben erwachsen der Sozialen Arbeit aus dieser Trias?

E rotik in der Sozialen Arbeit ist ein eher wenig bearbeitetes Feld in Nachschlagewerken und Fachpublikationen. In den gängigen Handbüchern und Lexika der Sozialen Arbeit finden sich Artikel zur Sexualität - zumeist im Sinne der Sexualerziehung respektive sexuellen Bildung oder der Auseinandersetzung mit sexueller Gewalt. Erotik existiert hingegen weder als Stichwort noch als Überschrift. Vielleicht erscheint die Thematisierung von Sexualität auch als erforderlicher, denn sie ist juristisch erlaubt oder verboten, sozial erwünscht oder unerwünscht.

\section{Erotik - Sexualität - Liebe}

Doch was ist mit Erotik? Ist sie noch anstößiger, da sie weniger auf spannungsabbauenden Vollzug als auf sehnsüchtiges Verlangen ausgerichtet ist? Laut Duden meint Erotik vor allem „den geistig-psychischen Bereich einbeziehende sinnliche Liebe“ (https://www.duden.de/ rechtschreibung/Erotik, Zugriff: 02.08.2021). Psychoanalytisch ausgedrückt handelt es sich um libidinöse Besetzungen, d. h. triebhaft-energetische Aufladungen im Sinne eines kulturell vermittelten, gefühlshaften Erlebens von Sinnlichkeit und Begehren (Brückner 2013). „Im Prinzip ist alles erotisierbar. So löst das Knie, das zu

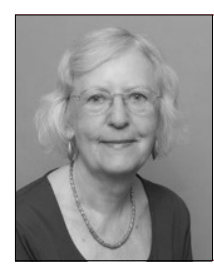

\section{Margrit Brückner}

Frankfurt University of Applied Sciences, Frankfurt, Deutschland

*1946, Dr., Professorin (i.R.), Soziologin, aktuelle Tätigkeit: Supervisorin, Fortbildnerin, Lehrbeauftragte. Wissenschaftliche Schwerpunkte: Frauen- und Geschlechterforschung.

brueckn@fb4.fra-uas.de

Zusammenfassung Zunächst werden Verhältnisse zwischen Erotik - Sexualität - Liebe ausgelotet. Anschließend geht es um historischen und gesellschaftlichen Wandel dieser Verhältnisse. Dann werden erotische Gehalte von Beziehungen in der Sozialen Arbeit und der professionelle Umgang mit ihnen diskutiert. Zuletzt gibt es einen Ausblick auf die notwendige Demokratisierung der zwischenmenschlichen Sphäre.

Schlüsselwörter Erotik, Sexualität, Liebe, Gesellschaftliche Rahmungen berühren einmal mit großer Spannung verbunden war, heute vielleicht überhaupt nichts mehr in uns aus. Begehren scheint also nomadisch und flüchtig. Zugleich ist die libidinöse Bedeutungsaufladung etwas Starkes, dem sich die Menschen, ähnlich wie der Verliebtheit, schwerlich entziehen können" (Eck 2018, S. 11). Das macht Erotik zu einem schwer greifbaren Thema, das uns eher individuell erscheint, auch wenn sich Kunst und Kultur zu allen Zeiten mit erotischen Darstellungen beschäftigt haben und unser jeweiliges Verständnis von Erotik prägen. Je nach Epoche und Milieuzugehörigkeit werden diese Darstellungen als Hochkultur oder als Pornografie wahrgenommen. Erotisch aufgeladener Kunstgenuss ist hier aber ebenso wenig Thema wie selbstbezogene Varianten der Erotik, sondern es soll um zwischenmenschliche Erotik gehen, da Soziale Arbeit mit Beziehungen zu tun hat.

\section{Zwischenmenschliches erotisches Erleben}

Erotisches Erleben bedarf der Selbstwahrnehmung als begehrendes und als begehrenswertes Wesen, was einen entsprechenden gesellschaftlichen Raum sowie die subjektive Ermöglichung solcher Empfindungen qua Sozialisationsprozess und Persönlichkeitsbildung voraussetzt. Sonst verbleibt Erotik - zumeist schicht- und geschlechtsspezifisch - beschränkt, weil nicht allen Gesellschaftsmitgliedern gleichermaßen ein Anrecht auf Begehren zugesprochen wird und diese es sich dann nur schwerlich aneignen können. Gelebte Erotik meint also eine innere und äußere Freiheit mit erregenden Gefühlen umzugehen, den eigenen Leib erotisch zu besetzen und erfordert ein spielerisches Moment, das es ermöglicht, Erregung gleichsam in der Schwebe zu halten und eine Umsetzung z. B. in sexuelle Praxis als Möglichkeit, nicht aber als zwangsläufig zu verstehen. „Sexualität braucht die ernsthafte Kommunikation während Erotik die spielerische Kommunikation nötig hat" (Mahnke und Sielert 2013, S. 147). Dieses spielerische Moment der Erotik eröffnet die Perspektive, sich der eigenen Lust, dem eigenen Verlangen und dem Anderer in der - relativen - Gewissheit auszusetzen, sich selbst und 


\section{Extrablick: Sexualität in der Sozialen Arbeit}

andere nicht zu gefährden durch Herabsetzung, Übergriffigkeit oder Selbstaufgabe (Benjamin 2020). Ohne diese Fähigkeit, sehnsüchtiges Verlangen und lustvolle Erregung als Empfindung zu spüren, gerät das Begehren schnell zur Triebabfuhr - die damit nicht per se herabgestuft werden soll, solange alle Beteiligten eine Konsensmöglichkeit haben. Erotik bedarf einer Demokratisierung der zwischenmenschlichen Sphäre (Giddens 2016), indem wir uns selbst und anderen je eigene erotische Gefühle zugestehen und mehr oder weniger schmerzlich genießen. Denn Erregung und Begehren drängt nach Erfüllung, endet aber auch damit.

\section{Einbindung von Erotik in kulturelle Rituale}

Deutlich wird die kulturelle Einbindung von Erotik in „Ritualen des Begehrens“ (Schetsche 2013, S. 234), wie wir sie z. B. beim Flirten, im Paartanz oder beim Dating finden, mit ihren Codes der Signalisierung von Interesse, Abwarten, Grenzziehung und Wieder-AufeinanderZugehen. Erst das kulturelle Wissen um die Bedeutung dieser Codes ermöglicht das Spiel, da diese Sicherheiten schaffen, dass die Annäherung (zumindest zunächst) ein Spiel bleibt, das ein Kribbeln auf der Haut verursacht, aber Rückzug für beide Seiten ohne Gesichtsverlust ermöglicht. Für das Tango-Tanzen konstatiert Nicole NauKlapijk: „Ohne diese Sicherheit [der Rituale] könnten die Tänzer sich nicht in aller Intensität dem Partner im Tanz hingeben. Sie wären zu Zurückhaltung gezwungen, denn sie müssten sich schützen vor der Bedeutung ihrer Geste im Tanz; dem Wunsch nach Nähe, der brodelnden Leidenschaft und Phantasie. Nur im Schutz der Rituale dürfen sie provozieren und fordern, sich hingeben und genießen, Leidenschaft und Sehnsucht empfinden und zum Ausdruck bringen“" (Nau-Klapijk 1999, S. 147/148 zit. nach Schetsche 2013, S. 241).

\section{Gesellschaftliche Rahmung erotischer Verhältnisse}

Erotik wird in unserer Gesellschaft zumeist als erotische Liebe in Form „serieller Monogamie“, d. h. der Abfolge zumeist - aber keineswegs ausschließlich - heterosexueller, weitgehend exklusiver Zweierbeziehungen gelebt. Das noch heute vorherrschende romantische Liebesideal ist im 18. Jahrhundert im Bürgertum als Teil der Aufklärung entstanden und bringt Erotik als leidenschaftliche Liebe und Ehe zusammen (Burkart 2019). Der Fortschritt dieses Ideals liegt in der Anerkennung der Individualität von Frau und Mann und ihrer jeweiligen Liebeswünsche, gleichzeitig wird die hierarchische Differenz zwischen den Geschlechtern fortgeschrieben: der Mann als Beschützer der Frau und als Erwecker weiblicher Sexualität. Daraus hat sich zunehmend ein partnerschaftliches, auf Gleichheit beruhendes Liebes- ideal entwickelt, ohne romantische Vorstellungen von Sehnsuchtserfüllung maßgeblich außer Kraft zu setzen. „Ohne die Orientierung an kulturellen Idealen - wie bspw. die romantische Liebe - aufzugeben, zeigen sich Paarbeziehungen gegenwärtig als weniger linear verlaufend, in ihren Grenzen diffuser, räumlich flexibler und zunehmend als praktische Leistung der Partner"innen“" (Wutzler und Klesse 2021, S. 8).

Besonders sozial benachteiligte Menschen aller Altersgruppen, Geschlechter und Herkünfte fühlen sich häufig traditionellen Vorstellungen von Weiblichkeit und Männlichkeit mit ihren hierarchisierten Rollenteilungen in Liebe, Sexualität und Erotik verbunden. Auch bevölkerungsweit sind in unserer diverser werdenden Gesellschaft traditionelle Arrangements in Paarbeziehungen weiterhin verbreitet, spätestens wenn Kinder geboren werden (Wimbauer und Motakef 2019). Die geschlechtsspezifischen Vorgaben traditioneller Geschlechterverhältnisse mit ihren Vereinseitigungen des Begehrens basieren nach dem Verständnis der Psychoanalyse auf unbewussten Wünschen und Ängsten (Willi 2012): Kernelement männlicher Identität ist Grenzziehung aus Angst vor Verschmelzung; weibliche Identität kreist hingegen um Durchlässigkeit von Ich-Grenzen zugunsten von Eins-Seins mit dem Partner. Damit werden die Geschlechter um die jeweiligen psychischen Fähigkeiten und sozialen Möglichkeiten des anderen Geschlechts gebracht, bezogen auf Erotik heißt das: Männer können sich nur schwer als Begehrte und Frauen sich nur schwer als Begehrende sehen und erleben.

Die dennoch wachsende Vielfalt der Liebesmöglichkeiten jenseits traditioneller Vorgaben schafft neue Freiheiten, aber auch Entscheidungsdruck und Auswahlnotwendigkeiten (wie will ich leben?) und damit tendenziell eine Rationalisierung von Liebe und Erotik (Stierlin 1997). Doch diese - nicht zuletzt auf Legalisierungen (z. B. 2017 Ehe für alle) beruhende - Ermöglichung von Vielfalt in Erotik, Liebe und Sexualität ist je nach Milieu und Lebenslage sehr unterschiedlich unangefochten lebbar. Interessant ist, dass Mädchen wie Jungen heute sexuell später aktiv werden als vor zehn Jahren (Jugendliche mit Migrationserfahrung noch etwas später) (BZgA 2020), weil sie die richtige Person noch nicht gefunden haben und/oder sich zu jung fühlen. Das spricht für eine wieder engere Verknüpfung von Sexualität, Liebe und Erotik - der öffentlichen Sexualisierung vieler Lebensbereiche zum Trotz.

\section{Verhältnisse zwischen Erotik und Sozialer Arbeit}

Gesellschaftlich geprägte Liebesverhältnisse, Erotik und Sexualität gehören zur Sozialen Arbeit, da sie professionell mit Lebenslagen und Lebensentwürfen von 
Menschen befasst ist und Menschen unterstützende Beziehungsangebote macht, ob in der Jugendarbeit, der Familienhilfe oder der Altenarbeit (Schmauch 2021). Traditionell beschäftigt sich Soziale Arbeit bis heute eher mit „sexueller Verwahrlosung“ insbesondere von Mädchen und jungen Frauen oder mit „sexueller Gefährdung" der Jugend als mit Erotik (Klein und Tuider 2017). Denn Soziale Arbeit hat weniger die gesellschaftliche Aufgabe zugeschrieben bekommen und übernommen, erotischer Lust zur Entfaltung zu verhelfen als Sexualität und Erotik vor allem der unteren Schichten einzudämmen. Dennoch gab es von Beginn professioneller Sozialer Arbeit an (vor allem von Sozialarbeiterinnen der ersten Frauenbewegung, Ende des 19. bis Anfang des 20. Jahrhunderts) eine kritische Auseinandersetzung mit der bürgerlichen Doppelmoral, die Frauen und Mädchen erst hilfebedürftig machte, indem sie Männern Rechte sexueller Ausbeutung gewährte. Heute ist eine professionelle Beschäftigung mit Liebe, Erotik, Sexualität auch wieder schwieriger als nach der „sexuellen Revolution“ der 1970er-Jahre, weil Grenzüberschreitungen, Gewalt und Ausbeutung nochmals in den Vordergrund gerückt sind (Brückner 2017). Die Auseinandersetzung mit sexualisierter Gewalt bleibt erforderlich, sollte aber nicht den Zugang zur Beschäftigung mit Erotik verstellen, denn alle Menschen haben sowohl ein Recht auf Gewaltfreiheit als auch auf Erotik und Sexualität.

Zwischenmenschliche Erotik bedarf der sexuellen Bildung zur Entfaltung von Imaginationen und der Befähigung zu Eigenschutz ebenso wie zur Respektierung der Wünsche und Grenzen des/der Anderen (Sielert 2014). Gerade für den gesellschaftlich schwächeren und körperlich verwundbareren Partner - in heterosexuellen Beziehungen oft die Frau - sind die Achtung von Wünschen und Grenzziehungen notwendig. Obgleich Erotik auch Überwältigung, Besitzergreifen und Hingabe, also Kontrollverlust meint, macht es doch einen Unterschied, ob das Verhältnis der Partner_innen zueinander geprägt ist vom Wissen um die Unveräußerlichkeit der Menschenwürde und körperliche Unversehrtheit für alle Beteiligten oder nicht.

\section{Erotische Aufladungen zwischen Klient_innen und Sozialarbeiter_innen}

Erotik und erotische Lebensformen von Klient_innen können sich für Sozialarbeiter_innen mit eigenen - oft der modernen Mittelschicht entlehnten - Vorstellungen von Gleichberechtigung und Abstimmungen decken, aber auch weit entfernt voneinander sein und entsprechend befremdlich wirken. Gerade die Arbeit mit jungen Frauen und Männern erfordert es, auf gegenseitiger
Anerkennung beruhende Vorstellungen von Liebe, Erotik und Sexualität zu vertreten, wenn Soziale Arbeit ihrem Anspruch einer Menschenrechtsprofession gerecht werden will - trotz des Wissens um eigene Brüche und mit einem entsprechend demütigen Blick auf die Brüche Anderer.

Eine der Voraussetzungen für professionelles Handeln ist die Auseinandersetzung mit eigenen erotisch-sexuellen Gefühlslagen. Ohne eigene Wünsche, Bedürftigkeiten und Schambereiche zu kennen, ist ein Verständnis sowohl für vertraute als auch für fremd empfundene Denk- und Verhaltensweisen von Klient_innen kaum möglich. Dazu gehört auch die Auseinandersetzung mit der eigenen Empfängnisbereitschaft für erotische Ausstrahlungen und Angebote. Altersunabhängig können sich Sozialarbeiter_innen von Klient_innen als auch umgekehrt Klient_innen von Sozialarbeiter_innen angezogen fühlen, wie in allen Berufsfeldern, in denen Professionelle auf Menschen treffen. Es geht nicht darum, erotische Anziehung zu verhindern - das wäre gar nicht möglich und würde zudem das Leben ärmer machen sondern darum, zu lernen, damit umzugehen. Auch wenn für Sozialarbeiter_innen das (keineswegs immer eingehaltene) Abstinenzgebot gilt, d. h. entsprechende Wünsche nicht auszuleben, ist es gleichwohl - insbesondere bezogen auf Kinder und Jugendliche - wichtig, erotische Angebote alters- und geschlechtsübergreifend zwar nicht zu erwidern, aber auch nicht zu verurteilen. Klient_innen sollten nicht beschämt oder herabgewürdigt werden, denn das würde ihr berechtigtes Bedürfnis nach Anerkennung als begehrenswertes Subjekt untergraben. Ob ein kleiner Flirt noch im Rahmen professionellen Handelns ist oder nicht, ist sowohl situationsabhängig als auch persönlichkeitsbedingt unterschiedlich. Immer ist jedoch die Frage der ungleichen Machtverhältnisse zu berücksichtigen und ihre Bedeutung für die Folgen des jeweiligen Verhaltens zu reflektieren. Ein angemessener Umgang mit erotisch aufgeladenen Begegnungen ist alles andere als einfach, daher bedarf es ausreichender professioneller Reflexionsräume und kollegialer Austauschmöglichkeiten.

\section{Erotik am Arbeitsplatz}

Überall, wo Menschen zusammenarbeiten, auch in Hochschulen oder Einrichtungen der Sozialen Arbeit, werden erotische Wünsche wach und gibt es erotische gefärbte Begegnungen (Schmauch 2021). Trotz des auf zweckrationales und zielorientiertes Handeln ausgerichteten Aufbaus von Organisationen spielt auch da Erotik eine (mehr oder weniger) geheime Rolle, denn erotische Begegnungen bedeuten Anerkennung von Gleichgesonnenen, bieten Ausgleich zur Arbeitsanstrengung und 


\section{Extrablick: Sexualität in der Sozialen Arbeit}

können die Arbeitsfreude steigern. „Erotische Beziehungen gehören damit zu den bestimmenden, aber nicht kontrollierbaren Einflussgrößen innerhalb der mikropolitischen Landschaft einer Organisation. Nicht nur der Reiz des Verbotenen macht Erotik in Organisationen so attraktiv. Wo immer Menschen zusammenkommen, finden sie sich auch erotisch anziehend, zumal in einer Umgebung, die die ganze Persönlichkeit fordert" (Saul-Soprun 1998, S. 73). Diese erotischen Begegnungen können von - beidseits gewünschten - kurzen Affären bis zu langfristigen Paarbeziehungen reichen.

Frauen müssen - so zeigt die Erfahrung - bei Affären aufgrund der hierarchisierten Geschlechterordnung eher um ihren Ruf fürchten als Männer. Problematisch können solche Beziehungen aller sexuellen Orientierungen auch sein, wenn Hierarchiegrenzen übersprungen und so Formen der Machtausübung möglich werden. Schwierig gestalten sich auch Beziehungsabbrüche, die auf Teams ausstrahlen. Teams können befürchten, dass sich das Paar gegenseitig bevorzugt oder gemeinsame Absprachen trifft. Somit haben erotische Begegnungen am Arbeitsplatz einerseits einen besonderen Reiz, andererseits bringen sie auch besondere Gefährdungen mit sich. Vor allem Frauen (s. „\#MeToo“) erfahren in der Arbeitswelt auch unerwünschte Annäherungen, die hier aber nicht Thema sind.

\section{Demokratisierung der zwischenmenschlichen Sphäre}

Gelebte Erotik und erotische Anziehung ist ein aufregendes Lebenselixier und nur begrenzt beherrsch- und steuerbar. Gerade das macht die Art und Weise, in der eine Gesellschaft Bilder des Erotischen produziert, aufnimmt, gutheißt oder verbietet für unser aller individuelle Entwicklungsmöglichkeiten und Bildungsprozesse bedeutsam. Geschlechterdemokratie im Sinne von Gleichwertigkeit aller Geschlechter und Flexibilisierung der Geschlechterrollen schafft Möglichkeiten, die mit Erotik einhergehende Verletzbarkeit durch gegenseitige Anerkennung einzubeziehen und an die nächste Generation weiterzugeben - auch in der professionellen Arbeit (bpb 2020). Mit der Menschenwürde vereinbare Bandbreiten kollektiver erotischer Bilder und individueller erotischer Praxen sollten immer wieder neu ausgelotet werden, da sich mit den jeweiligen gesellschaftlichen Rahmungen auch Vorstellungen menschlicher Würde wandeln. Sie unterliegen Bildungsprozessen und Positionierungen, die die Soziale Arbeit mitgestalten sollte, wenn sie ihrem Anspruch, Lebensbewältigung in Hinblick auf ein gelingenderes Leben zu unterstützen (Thiersch 2020), gerecht werden will, denn Liebe, Sexualität und Erotik gehören unabdingbar dazu. Nur eine aktive Gestaltung ermöglicht einen haltenden Denkund Handlungsrahmen, auf den sich dann Sozialarbeiter_innen beziehen können. Zusammenfassend bedeutet Demokratisierung der zwischenmenschlichen Sphäre bezogen auf Liebe, Sexualität und Erotik:

- eine soziale Ordnung mit gleichen Lebens- und Liebenschancen für alle Geschlechter und alle sexuellen Lebensweisen,

- eine kulturelle Ordnung auf der Basis von Vielfalt auch in Liebesdingen,

- persönliche Entwicklungschancen auch bezogen auf ein erotisches Selbst- und Weltverständnis.

Eingegangen. 30. September 2021

Angenommen. 29. November 2021

Funding. Open Access funding enabled and organized by Projekt DEAL.

Open Access. Dieser Artikel wird unter der Creative Commons Namensnennung 4.0 International Lizenz veröffentlicht, welche die Nutzung, Vervielfältigung, Bearbeitung, Verbreitung und Wiedergabe in jeglichem Medium und Format erlaubt, sofern Sie den/die ursprünglichen Autor(en) und die Quelle ordnungsgemäß nennen, einen Link zur Creative Commons Lizenz beifügen und angeben, ob Änderungen vorgenommen wurden.

Die in diesem Artikel enthaltenen Bilder und sonstiges Drittmaterial unterliegen ebenfalls der genannten Creative Commons Lizenz, sofern sich aus der Abbildungslegende nichts anderes ergibt. Sofern das betreffende Material nicht unter der genannten Creative Commons Lizenz steht und die betreffende Handlung nicht nach gesetzlichen Vorschriften erlaubt ist, ist für die oben aufgeführten Weiterverwendungen des Materials die Einwilligung des jeweiligen Rechteinhabers einzuholen.

Weitere Details zur Lizenz entnehmen Sie bitte der Lizenzinformation auf http://creativecommons.org/licenses/by/4.0/deed.de.

\section{Literatur}

Benjamin, J. (2020). Die Fesseln der Liebe. Psychoanalyse, Feminismus und das Problem der Macht (5. Aufl.). Frankfurt a. M.: Klostermann. Nachdruck

bpb (2020). Geschlechterdemokratie. Informationen zur politischen Bildung, Bd. Nr. 342. Bonn: bpb.

Brückner, M. (2013). Amalgamierungen des Begehrens: Körperliche Lust, erotisches Wünschen, psychosoziale Einpassungen. In R.-B. Schmidt \& U. Sielert (Hrsg.), Handbuch Sexualpädagogik und sexuelle Bildung (S. 220233). Weinheim, München: Juventa.

Brückner, M. (2017). Brüche und Kontinuitäten: Verhältnisse zwischen Liebe/Sexualität und Sozialer Arbeit. In A. Klein \& E. Tuider (Hrsg.), Sexualität und Soziale Arbeit (S. 37-56). Baltmannsweiler: Schneider Hohengehren.

Burkart, G. (2019). Liebe: historische Formen und theoretische Zugänge. In B. Kortendiek, B. Riegraf \& K. Sabisch (Hrsg.), Handbuch interdisziplinäre Geschlechterforschung (Bd. 2, S. 1093-1102). Wiesbaden: Springer.

BZgA (2020). Jugendsexualität 9. Welle. https://www.forschung.sexualaufklaerung.de/service/nachrichten-und-statistiken-archiv. Zugegriffen: 14. Juli 2021. 
Eck, A. (2018). Einleitung. In A. Eck (Hrsg.), Der erotische Raum. Fragen der weiblichen Sexualität in der Therapie. Heidelberg: Carl Auer.

Giddens, A. (2016). Wandel der Intimität. Sexualität, Liebe und Erotik in modernen Gesellschaften. Frankfurt: Fischer.

Klein, A., \& Tuider, E. (Hrsg.). (2017). Sexualität und Soziale Arbeit. Baltmannsweiler: Schneider Verlag Hohengehren.

Mahnke, E., \& Sielert, U. (2013). Die Kunst des Scheiterns und Gelingen in Lust und Liebe. In R.-B. Schmidt \& U. Sielert (Hrsg.), Handbuch Sexualpädagogik und sexuelle Bildung (S. 141-154). Weinheim, München: Juventa.

Saul-Soprun, G. (1998). Erotik. In P. Heinrich \& J. Schulz zur Wiesch (Hrsg.), Wörterbuch der Mikropolitik (S. 73-75). Wiesbaden: Springer.

Schetsche, M. (2013). Rituale des Begehrens. In R.-B. Schmidt \& U. Sielert (Hrsg.), Handbuch Sexualpädagogik und sexuelle Bildung (S. 234244). Weinheim, München: Juventa.

Schmauch, U. (2021). Sexualität in der Ausbildung der Sozialen Arbeit. In D. Höblich \& D. Mantey (Hrsg.), Handbuch Sexualität und Soziale Arbeit. Weinheim, Basel: Beltz Juventa. erscheint demnächst.

Sielert, U. (2014). Sexualerziehung, sexuelle Bildung und Entwicklung von Sexualkultur als sozialpädagogische Herausforderung. Sozialmagazin, 39(1-2), 38-45.

Stierlin, H. (1997). Verrechnungsnotstände: Über Gerechtigkeit in sich wandelnden Beziehungen. Familiendynamik, 22(2), 136-155.

Thiersch, H. (2020). Perspektiven des Professionskonzepts der Sozialen Arbeit. In Bielefelder Arbeitsgruppe 8 (Hrsg.), Wie geht es weiter mit Sozialer Arbeit? (Bd. 8, S. 32-43). Lahnstein: neue praxis.

Willi, J. (2012). Die Zweierbeziehung. Das unbewusste Zusammenspiel von Partnern als Kollusion. Reinbek: rororo.

Wimbauer, C., \& Motakef, M. (2019). Paarbeziehungen: Paare und Ungleichheiten als Gegenstand der Geschlechterforschung. In B. Kortendiek, B. Riegraf \& K. Sabisch (Hrsg.), Handbuch interdisziplinäre Geschlechterforschung (Bd. 2, S. 1103-1110). Wiesbaden: Springer.

Wutzler, M., \& Klesse, J. (Hrsg.). (2021). Paarbeziehungen heute: Kontinuität und Wandel. Weinheim: Beltz, Juventa.

Hier steht eine Anzeige. Springer 